\title{
LA NUEVA RUTA DE LA SEDA Y LA DIPLOMACIA INTERNACIONAL DE REGOCIOS
}

\author{
neu Silk Road and International Business \\ Diplomacy
}

\author{
Francisco Valderrey Villar \\ Instituto Tecnológico y de Estudios Superiores de Monterrey \\ E-mail: francisco.valderrey@itesm.mx \\ Daniel Lemus Delgado \\ Instituto Tecnológico y de Estudios Superiores de Monterrey \\ E-mail: dlemus@itesm.mx
}

En el presente trabajo se analiza la diplomacia internacional de negocios en el contexto de la Nueva Ruta de la Seda, la iniciativa geoeconómica más importante del gobierno chino. Se parte del supuesto teórico de la interdependencia compleja de Kohane y Nye, para señalar la magnitud de los obstáculos que se deberán solventar. Asimismo, se plantea como mecanismo complementario la participación directa de actores no estatales en la resolución de los conflictos y se sugiere que las empresas chinas con una participación en los proyectos de la Nueva Ruta de la Seda intervengan directamente en defensa de sus propios intereses comerciales, sin depender de su gobierno o de la diplomacia tradicional. Se debe recurrir a la diplomacia internacional de negocios para acercar las posiciones de las partes involucradas y aumentar las probabilidades de éxito del proyecto.

China; interdependencia compleja; diplomacia internacional de negocios; Nueva Ruta de la Seda.

Q Key words China; complex interdependence theory; international business diplomacy; New Silk Road. 
This article seeks to analyze International Business Diplomacy within the context of the New Silk Road, the largest geoeconomic initiative from the Chinese government. The magnitude of the obstacles faced by the Chinese government is discussed, under the theorethical framework of complex interdependence proposed by Kohane and Nye. Additionally, direct participation of non-state actors is proposed as an alternative for conflict resolution on the different issues that may arise. Specifically, Chinese enterprises with a substantial involvement in the different projects are encouraged to protect their own comercial interests, without relying on their own government or from traditional diplomacy. Those mechanisms are insufficient, in lieu of the magnitude of the project and the multiplicity of actors; therefore, international business diplomacy will be necessary to embrace stakeholders and improve the probabilities of success of the entire project.

\section{Introducción}

En el año 2013, el presidente chino Xi Jinping, inspirándose en la legendaria Ruta de la Seda, anunció un ambicioso proyecto para impulsar el comercio internacional, los flujos de inversión y el intercambio cultural entre China y los países de Asia central, Europa del este y África oriental. El punto de partida de este proyecto es la construcción de una extensa y sólida red de infraestructura, bajo generosos esquemas de financiamiento chino. Como se señaló en el plan de acción de este proyecto, la intención final es conectar al vibrante círculo de economías de Asia oriental con las economías desarrolladas de Europa y con un grupo de países de África y Asia central que cuentan con un enorme potencial para su desarrollo económico (National Development and Reform Commission, 2015).

El nombre de la iniciativa se acuñó recurriendo a una expresión típicamente china, Yi Lu Yi Dai (一带一路) ${ }^{1}$. La iniciativa, conocida como la Nueva Ruta de la Seda, busca satisfacer múltiples objetivos, tanto económicos, como políticos y sociales. Entre estos objetivos, tal vez el de mayor alcance sea el de reafirmar el ascenso de China y su creciente influencia en el escenario internacional. Por ende, esta iniciativa incide en la manera de concebir de manera diferenciada un "gobierno global", resultado del esfuerzo sistemático de la élite burocrática china para desarrollar un nuevo orden internacional que modifique el statu quo del sistema internacional (Beeson y Li, 2016).

La idea fue concebida con el propósito de que en el futuro la Nueva Ruta de la Seda sea incluyente respecto a la incorporación de nuevos países, pero siempre bajo el liderazgo del gobierno chino. La importancia de esta iniciativa quedó demostrada tras el anuncio realizado en Kazajstán en el año 2013 de la primera parte del plan, la "franja económica de la ruta de la seda”. La segunda parte del plan se llevó a cabo en Indonesia, donde se informó sobre el nuevo eje marítimo (Bhoothalingam, 2016). Ambas rutas incluyen países de escaso volumen comercial con China, pero con potencial para desarrollarlo, como ocurre con algunas na-

1 Este término ha sido inicialmente traducido al inglés como Belt and Road. En la literatura sobre el tema se usan diferentes acrónimos, como OBOR (One Belt One Road) o B\&R, por ejemplo, pero sigue sin definirse con precisión el alcance de la misma. La traducción al castellano que proponen las fuentes oficiales del gobierno chino, "Franja y Ruta”, no parece ser muy afortunada, puesto que ni evoca las maravillas de la ruta histórica de la seda, ni tampoco comunica el sentido original de interacción de naciones colaborando para generar un cinturón de prosperidad a través de Eurasia. Pese a que el proyecto recibe diferentes nombres, nosotros usaremos la expresión más común en castellano, la Nueva Ruta de la Seda.

\section{La iniciativa, conocida como la nueva Ruta de la Seda, busca satisfacer múltiples objetivos, tanto económicos, como políticos y sociales}


ciones de África oriental (Naqvi, 2015). En ambos casos se privilegia el rol del Estado en la construcción de una infraestructura que permita incrementar los flujos comerciales, financieros y culturales entre países.

Sin embargo, pese al notorio peso del gobierno chino, tanto en el planteamiento inicial como en su posterior desarrollo, es evidente la necesidad de una activa participación de actores no estatales para que este proyecto sea concluido de manera efectiva. Por lo tanto, nosotros proponemos que la iniciativa de la Nueva Ruta de la Seda debe ser analizada bajo modelos teóricos que vayan más allá de una visión estatocéntrica. Así, empleamos un enfoque basado en el supuesto teórico de la interdependencia compleja de Kohane y Nye, lo que permite contar con un nivel de análisis de mayor profundidad y reconoce la importancia de actores no estatales en este proceso. Mediante este enfoque, buscamos contribuir a la mejor comprensión de la política exterior china, que va más allá de una diplomacia tradicional.

Aunque muchos entes diferentes adquieren importancia en este contexto, destacamos el papel de las empresas multinacionales, en particular las de China, ya que estas llevarán a cabo los ingentes proyectos que se incluyen en esta iniciativa y, presumiblemente, deberán interactuar con otros actores, además de los gobiernos locales donde desarrollen sus actividades. En este escenario, sumamente complejo, proponemos que la diplomacia internacional de negocios puede ser el medio que ofrezca a las empresas los mecanismos de resolución de conflictos más adecuados a la realidad de sus operaciones en el exterior.

El presente artículo tiene como objetivo demostrar la importancia de la diplomacia internacional de negocios, especialmente en escenarios de gran complejidad, en el que se ven involucradas entidades privadas. En primer lugar, relacionaremos el proyecto de la Nueva Ruta de la Seda con el marco paradigmático de la interdependencia compleja. En segundo lugar, describiremos las características de la diplomacia internacional de negocios, sus elementos distintivos respecto a otros modelos de diplomacia y destacaremos su importancia en el contexto de la Nueva Ruta de la Seda. En tercer lugar, analizaremos el rol de la diplomacia internacional de negocios como elemento fundamental para superar los desafíos del proyecto que nos ocupa. En definitiva, veremos la aplicabilidad de la teoría de la Interdependencia Compleja, así como la aportación de la diplomacia internacional de negocios como elemento complementario que puede coadyuvar al éxito de este proyecto y a aquellos que se destacan por la complejidad de intereses interdependientes.

\section{La nueva Ruta de la Seda}

El concepto en sí mismo de la Nueva Ruta de la Seda no es de fácil comprensión: bajo la alusión a la tradicional ruta usada por mercaderes para transportar la seda y otros productos de lujo desde Asia hasta el continente europeo, se presentan en realidad, dos ejes diferentes, quedando además implícitos un sinnúmero de propósitos. Las rutas propuestas están histórica y geográficamente diferenciadas y son el fruto de dos proyectos diferentes, tal y como se plasma en el mapa 1. El primero, Silk Route Economic Belt (Franja Económica de la Ruta de la Seda) se basa en el desarrollo de un cinturón económico que se extiende por vía terrestre desde el oeste y el interior de China hacia Europa del este, a través de Asia central. Este es el proyecto más destacado y tiene su antecedente histórico en las diferentes rutas de la seda que alcanzaron su apogeo durante la dinastía Tang, entre los años 618 y 906 de nuestra era (Hansen, 2015). 
La segunda ruta, XXI Century Maritime New Silk Road, (Ruta Marítima del Siglo XXI de la Ruta de la Seda) pretende revivir las rutas comerciales que impulsaban por mar el comercio de la seda. Estas rutas servían para unir las costas de China con lugares tan remotos como las costas africanas del Océano Índico. En el trayecto se incorporaban las especias y otros productos de la India y el Sudeste Asiático. Esta ruta floreció principalmente durante la dinastía Ming, cuando bajo el mando del almirante Zheng He, distintos viajes marítimos no solamente llevaron productos chinos a larga distancia, sino que también dieron a conocer la superioridad cultural de China (Menzies, 2008). Se sugiere, por tanto, considerar ambos proyectos dentro de una misma iniciativa, la Nueva Ruta de la Seda, pero dándole un sentido más amplio, puesto que este proyecto incluye elementos de avance económico, de estrategia y de visión nacional (Van der Leer y Yau, 2016).

Mapa 1: mapa de la nueva Ruta de la Seda

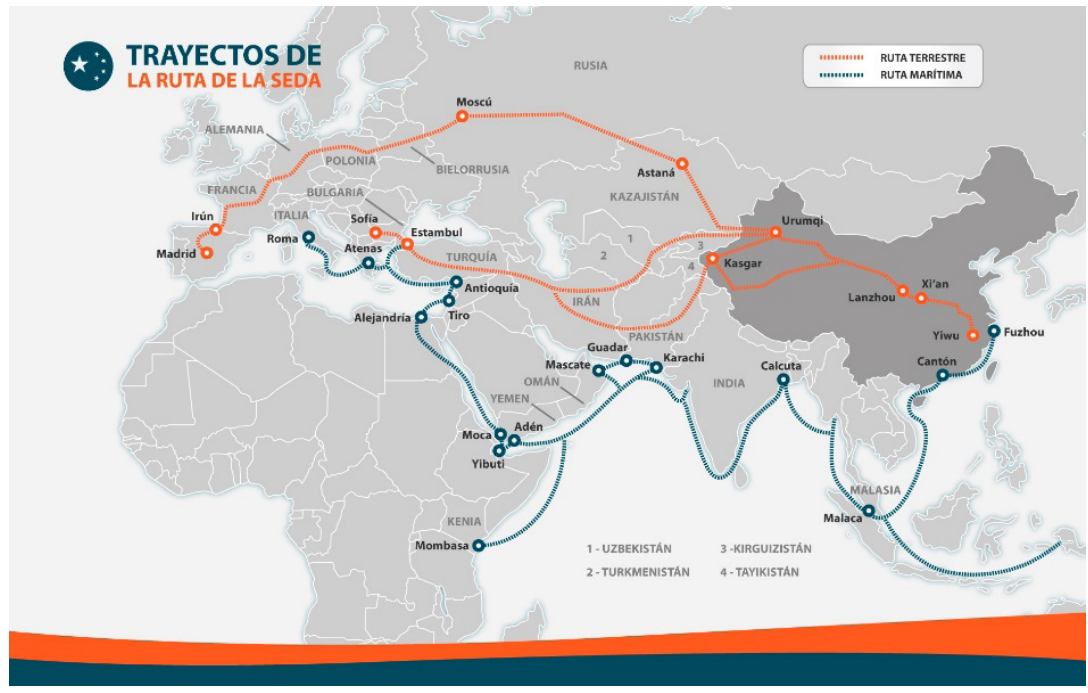

Fuente: http://www.esglobal.org

La iniciativa, en su conjunto, es parte de la estrategia de desarrollo de China, en su búsqueda para profundizar sus vínculos económicos con países de Asia, Europa y África. En su conceptualización, la iniciativa tiene el potencial para vincular a sesenta países, con la posibilidad de extenderse sin un límite marcado por las tradiciones o la geografía, en los que se asienta una población de más de cuatro mil millones de personas (Lee, 2016).

Así, la Nueva Ruta de la Seda se constituye en un medio privilegiado para alcanzar el "sueño chino”, la principal propuesta política que Xi Jinping lanzó al inicio de su mandato. Conforme al presidente, "el sueño de China es el significado interno de cumplimiento y el desarrollo del socialismo con características chinas" y su esencia se basa en transformar a la nación en un país pudiente, mediante la revitalización del país y con el objetivo final de incrementar el bienestar de su población (Ferdinand, 2016, p. 946).

En este plan, como lo ha advertido Lo (2015) pueden verse tres grandes objetivos de largo plazo:

a. El desarrollo de infraestructura regional.

b. La exportación de excedentes de producción.

c. Y la mejora de China en sus relaciones comerciales internacionales. 
En primer lugar, la implementación de esta estrategia debe detonar el desarrollo de la infraestructura regional, a través de la conexión de este país con Asia, Europa y África, permitiendo impulsar al unísono la internacionalización de su moneda, el renminbi, mediante el fomento de su uso en las transacciones comerciales y financieras. En segundo lugar, ya en un plano interno, se busca que China pueda de alguna forma exportar el exceso de capacidad productiva que ha generado en los últimos años, con la consiguiente mejora en la rentabilidad de sus inversiones y en la estabilidad de su crecimiento económico. Como tercer objetivo, este proyecto debe servir para mejorar las relaciones de comercio bilateral y multilateral, puesto que el país ha quedado excluido de acuerdos comerciales importantes (Lo, 2015).

La perspectiva oficial del gobierno chino aúna tales elementos, aunque declarando que el proyecto de la Nueva Ruta de la Seda tiene como objetivo primordial la promoción de la conectividad entre los continentes de Asia, Europa y África y sus mares adyacentes. Explica, además, que se busca establecer y fortalecer las alianzas entre los países a lo largo de estos corredores geográficos, con la intención de fomentar un desarrollo sostenible, independiente y equilibrado entre esas naciones. De esta manera, se pretende que los proyectos de conectividad de esta iniciativa satisfagan múltiples propósitos. Entre estos destacan: contribuir a alinear y coordinar las estrategias de desarrollo de los países involucrados en el proyecto, impulsar el potencial del mercado en esta región, promover la inversión y el consumo, crear demandas y oportunidades de empleo, mejorar el nivel de vida de las personas y fomentar los intercambios culturales y el aprendizaje mutuo entre los pueblos de los países que abarca el proyecto. Se espera, siempre conforme al discurso oficial, que esta iniciativa sirva para generar un clima de comprensión, confianza y respeto mutuo que permita la vida en armonía, paz y prosperidad. Como telón de fondo se establece el compromiso de China, dentro de sus posibilidades, de asumir más responsabilidades y obligaciones en el escenario internacional y a realizar mayores contribuciones a la paz y al desarrollo de la humanidad (National Development and Reform Commission, 2015).

La ruta abarca más de sesenta países en una de las áreas del planeta con mayor auge económico, como demuestra el paulatino desplazamiento del centro de gravedad económico hacia el este (Olier, 2016), lo que también augura una creciente importancia geopolítica de esta zona y, en general, de la cuenca del Pacífico. Su propósito va más allá de la construcción de redes físicas y vínculos materiales, expandiéndose a diferentes ámbitos y haciendo evidente la inclusión de propuestas multisectoriales.

Ahora bien, la iniciativa de la Nueva Ruta de la Seda ha sido estudiada desde distintas perspectivas, aunque el denominador común ha sido el análisis teórico que refleja, en mayor o menor medida, una postura estatocéntrica. Conforme a la visión de Zhao (2015), este proyecto parte de una serie de programas y campañas de diplomacia pública que el gobierno chino ha puesto en marcha desde el año 2013, con la intención de ganarse el respeto, tanto de la comunidad internacional como de sus propios ciudadanos, como parte de una búsqueda general de legitimidad política. Por lo tanto, puede decirse que la Nueva Ruta de la Seda es un instrumento que permite a China presentarse como una potencia emergente, logrando al mismo tiempo rejuvenecer sus valores y su sistema político. Por su parte, Yongjin Zhang (2016) considera que el dinamismo, un rasgo distintivo de la política exterior china bajo Xi Jinping, ha impulsado el surgimiento de esta iniciativa, reflejando en ello el activismo multidimensional y el impulso de la economía regional y global que el gobierno lleva a cabo. La iniciativa puede ser interpretada también como un intento de combinar las aspiraciones personales del presidente Xi con los anhelos del pueblo chino para recuperar el orgullo nacional y mejorar el bienestar social (Lo,

\section{La nueva Ruta de la Seda tiene como objetivo primordial la promoción de la conectividad entre los continentes de Asia, Europa y África y sus mares adyacentes}


2015). Nosotros sugerimos adoptar un enfoque teórico que supere los límites estrechos de la visión estatocéntrica de esta iniciativa.

\section{Los límites de la diplomacia tradicional en un mundo caracterizado por la interdependencia compleja}

Es fácil entender la trascendencia económica de la Nueva Ruta de la Seda, pero existen motivaciones geopolíticas que la tornan en un proyecto de gran impacto en la comunidad internacional. En efecto, mientras se desarrolla este proyecto, se está fraguando el acuerdo conocido como TPP (Trans Pacific Partnership) y que parece ser la némesis del plan liderado por China. El acuerdo, liderado de facto por Estados Unidos, está pendiente de ratificación por parte de los demás países signatarios, pero ya está fomentando una dualidad de bloques comerciales, operando en gran parte en el continente asiático. En este sentido debe también analizarse el impacto de ambas iniciativas, puesto que parecen responder en gran medida a la lucha hegemónica entre ambas potencias y los países que se decantan por uno u otro bloque comercial. Diferentes voces hacen hincapié en la importancia de que China no caiga en actitudes excluyentes y abra también oportunidades para empresas de Europa (Esteban y Otero-Iglesias, 2016), o incluso de otros continentes. En sí, la Nueva Ruta de la Seda debe analizarse como proyecto de enorme trascendencia para la estabilidad mundial; nosotros lo hacemos, apoyándonos en la teoría de la interdependencia compleja, tal y como se describe a continuación.

\subsection{La teoría de la interdependencia compleja}

Al inicio del siglo XXI, Robert Keohane y Joseph Nye publicaron un artículo en el que reflexionaban sobre la dinámica de la globalización. En el documento se enfatizaba la idea de que los actuales procesos de integración se acercan cada vez más al tipo ideal que ellos mismos habían propuesto veinte años antes, bajo el término de la interdependencia compleja (Keohane y Nye, 2000). Desde su perspectiva, el contenido de la política mundial está cambiando profundamente, aunque es probable que el sistema de Estados soberanos continúe siendo la estructura dominante en el sistema internacional. Los mismos autores afirmaban al inicio de la década de 1970 que: "la política mundial se está transformando, pero nuestros paradigmas conceptuales no han avanzado al mismo ritmo" (Keohane y Nye, 1973, p. 371). Dichas transformaciones desafiaban los supuestos básicos del análisis tradicional realista que postulaba que la anarquía y el dilema de seguridad al que tienen que hacer frente los Estados conducen inevitablemente al conflicto.

Para analizar la naturaleza de las transformaciones del escenario internacional, dichos autores propusieron el concepto de las relaciones transnacionales, el cual socava la centralidad del Estado como la unidad de análisis. Este concepto sostiene que, si bien el Estado es un actor unitario, las instituciones pueden superar los obstáculos a la cooperación que se derivan de la anarquía. Así, teóricamente, se propuso una forma de analizar la política internacional que superara los estrechos márgenes de la visión estatocéntrica del escenario internacional. A esta conceptualización teórica se le denominó interdependencia compleja.

Las principales características de la interdependencia compleja son: 
a) Presencia de canales múltiples entre sociedades.

b) Ausencia de una jerarquía de los temas de la agenda internacional.

c) Y el cambio en la trascendencia de la fuerza militar.

En primer lugar, se hace patente la presencia de canales múltiples que conectan a las sociedades, más allá de los vínculos forjados al amparo de los Estados nación. Estos canales incluyen: nexos informales entre élites gubernamentales, acuerdos formales entre agencias de servicio exterior y nexos informales entre élites no gubernamentales y organizaciones transnacionales. A partir de estos canales se llevan a la práctica relaciones interestatales, transgubernamentales y transnacionales. En segundo lugar, la agenda de las relaciones interestatales consiste en múltiples temas que no están determinados por una jerarquía clara o sólida. Esta ausencia de jerarquía implica que la seguridad militar no domina consistentemente la agenda, y en consecuencia muchos de los temas que prevalecen son resultado de la política interna de las naciones. Además, no existe una diferenciación clara entre los temas internos y externos de los Estados. En tercer lugar, la fuerza militar no es empleada por los gobiernos contra otros gobiernos de la región cuando predomina la interdependencia compleja. De esta manera, la fuerza militar resulta irrelevante para resolver discrepancias sobre aspectos económicos entre los miembros de una alianza, aunque puede no perder su importancia en las relaciones políticas y militares de esa alianza con un bloque rival.

Si aceptamos que la propuesta teórica descrita es válida para comprender las relaciones entre China y los países involucrados en el proyecto de la Nueva Ruta de la Seda, es posible sostener que el gobierno de ese país está utilizando su poderío económico para ganar influencia en la región y empezar a dibujar un escenario internacional que se ajuste mejor a sus propios intereses. Lo hace a través de la promoción del multilateralismo y el fomento de nuevos organismos internacionales regionales que ayuden al proyecto, como en el caso del Banco Asiático de Inversión en Infraestructura y la Organización de Cooperación de Shanghái. Sin embargo, tal y como defiende la propuesta de la interdependencia compleja, el esfuerzo de la élite burocrática china no es suficiente para alcanzar las metas trazadas debido a que el Estado chino no puede ser el único actor responsable en esta iniciativa.

Es evidente que nuevos actores tienen cada vez más peso en el escenario internacional, como ocurre con las empresas de ese país con proyección internacional, pues son la punta de lanza en el desarrollo de los proyectos de la Nueva Ruta de la Seda y los primeros en lucrarse de sus grandes beneficios comerciales y financieros. El proceso, sin embargo, es dinámico y bidireccional. Es decir, por una parte, estas empresas se convierten en instrumentos para implementar la política exterior de China en el exterior, mientras que por otra parte despliegan un activismo interno para conseguir situaciones de privilegio en los diferentes proyectos. De este modo, el actuar de las empresas internacionales involucradas en esta iniciativa es moldeado por la política exterior china, a la que al mismo tiempo también moldea.

\subsection{El enfoque de la diplomacia internacional de negocios}

Puede afirmarse que, a lo largo de la historia, la diplomacia ha establecido con éxito las reglas para las negociaciones, ha creado protocolos para llevar a cabo conversaciones tendentes a mantener la paz y ha tenido un impacto significativo en el avance de los acuerdos comerciales. Sin embargo, como postula Rasmussen (2016), la diplomacia es un concepto "esponja", que 
absorbe muchos significados, hasta el punto de que el concepto pierde mucho de su sentido original. En una concepción amplia de este término, se puede establecer que la diplomacia es un sistema de comunicación entre extraños; al igual que el diálogo a partir del cual se construye, la diplomacia trata de mediar a través del uso de la persuasión y la fuerza, de promesas y amenazas, de códigos y símbolos (Der Derian, 2001). No obstante, en un sentido más limitado, se puede establecer que la diplomacia se refiere a la conducción de las relaciones internacionales a través de los funcionarios del Estado que tratan de asegurar los objetivos de su política exterior por medio de la negociación, sin recurrir por tanto a la fuerza, la propaganda, o la ley (Berridge, 2002).

Aunque el inicio de la diplomacia moderna se remonta a la creación del sistema internacional derivado de los tratados conocidos como la Paz de Westfalia, es posible afirmar que la diplomacia como institución está experimentando una rápida transformación; lo hace en términos de los actores involucrados, de las prácticas específicas de lo que ha sido conceptualizado y en la pluralización de la diplomacia (Rasmussen, 2016). Hoy en día, la multiplicidad de actores internacionales no-estatales y, sobre todo, el aumento de su poder de influencia, ha obligado a redefinir la actividad propia de la diplomacia (La Porte, 2016). Por lo tanto, la diplomacia no tradicional juega un rol cada vez más importante en un mundo en el que cada día aumenta la interdependencia compleja entre los actores que lo conforman.

En la tabla 1 recogemos diferentes formas de diplomacia; no pretendemos que sea una tipología completa, pero de ella se desprende la creciente importancia de sus nuevas formas y del interés en las relaciones que involucran a nuevas entidades.

Tabla 1

\begin{tabular}{l|l|}
\hline Forma de diplomacia & Descripción \\
\hline Tradicional & $\begin{array}{l}\text { Se ocupa de asuntos que conciernen a Estados soberanos y organizaciones internacionales, } \\
\text { mediante representantes oficiales y bajo la coordinación de ministerios de asuntos exteriores }\end{array}$ \\
\hline Pública & $\begin{array}{l}\text { Se dirige a públicos de otras naciones para intentar modificar las percepciones o los intereses } \\
\text { de la nación en otros países }\end{array}$ \\
\hline Económica & $\begin{array}{l}\text { Defiende propósitos ideológicos o intereses geoeconómicos, a través de políticas, estrategias o } \\
\text { acciones que involucran decisiones económicas y financieras }\end{array}$ \\
\hline Comercial & $\begin{array}{l}\text { Es el trabajo de representantes oficiales en el servicio diplomático que llevan a cabo acciones } \\
\text { en apoyo de intereses comerciales y de negocios }\end{array}$ \\
\hline Corporativa & $\begin{array}{l}\text { Es el proceso que ayuda a establecer el poder y la legitimidad de las empresas, aunque no } \\
\text { necesariamente tiene un componente internacional }\end{array}$ \\
\hline Involucra el establecimiento y el mantenimiento de relaciones positivas por parte de empresas \\
\hline con representantes de gobiernos extranjeros y otros actores no gubernamentales, con el \\
propósito de construir y sostener su legitimidad en contextos de negocios internacionales
\end{tabular}

Fuente: adaptado de Valderrey, F. J. (2016). International Business Diplomacy: Lessons from Latin America. En Multinational Enterprise Management Strategies in Developing Countries. Hershey, PA: IGI Global

Debido a que la interdependencia compleja no es solamente política sino también económica, la diplomacia desempeña un papel crucial para impulsar los objetivos económicos de los actores internacionales. Desde la perspectiva de las metas económicas que se persiguen en un mundo 
interdependiente, se pueden establecer tres tipos de diplomacia: la económica, la comercial y la internacional de negocios. La primera forma incluye las actividades que los gobiernos realizan para favorecer sus intereses ideológicos o geoeconómicos, mediante lineamientos políticos, estrategias o acciones que involucran decisiones económicas y financieras. La segunda forma obedece a la defensa de intereses comerciales. Por lo general, estas actividades son el resultado de las interacciones entre naciones a través de una amplia gama de acciones de entidades semipermanentes y de representaciones internacionales. Ejemplo de lo anterior es la infraestructura de embajadas, consulados y otras instalaciones auxiliares a las empresas del sector público, complementada por actividades bilaterales diplomáticas, como ocurre con visitas comerciales o estatales. Por último, la diplomacia internacional de negocios busca establecer y mantener relaciones positivas con los representantes de gobiernos extranjeros y las partes interesadas no gubernamentales, con el objetivo de construir y sostener la legitimidad en un entorno empresarial extranjero (Wolters, 2012).

Sin lugar a dudas, la diplomacia internacional de negocios se acerca más a la realidad de las empresas multinacionales establecidas fuera de su país de origen, lo que ayuda en la solución de conflictos inmediatos con actores extranjeros, aunque también persigue fines diferentes en el largo plazo. Así, la diplomacia internacional de negocios consiste en el establecimiento, por parte de las empresas, de relaciones positivas con representantes de gobiernos extranjeros y las partes interesadas no gubernamentales, en temas económicos y no económicos, con el objetivo de construir y sostener la legitimidad, es decir salvaguardar la imagen corporativa y la reputación, en un ambiente extranjero de negocios (Wolters, 2012). Este tipo de diplomacia es supuestamente inherente a las actividades de todos los empleados de una organización que trabaja en el extranjero y de aquellos cuyas decisiones pueden afectar a las relaciones a través de fronteras. Sin embargo, la responsabilidad principal recae en los ejecutivos de alto nivel, cuya posición de liderazgo les obliga a estar analizando el entorno de negocios para detectar las amenazas y oportunidades para su organización (Saner, Yiu, y Sondergaard, 2000), aunque igualmente les confiere la autoridad para mediar en los conflictos que sobrepasan el interés inmediato de su empresa (Abbas, 2009). El tamaño de la organización que lleva a cabo acciones de diplomacia internacional es importante, ya que entre las empresas multinacionales de mayor tamaño se reconoce su importancia, cosa que no ocurre al nivel de las PYME (Ruël, 2013).

El concepto de la diplomacia internacional de negocios incluye cuatro áreas diferentes:

a) En el interior de las empresas.

b) Entre empresas.

c) En las relaciones entre la empresa y el gobierno.

d) En la arena de los negocios internacionales.

Por lo tanto: el primer ámbito de la diplomacia internacional de negocios se lleva a cabo dentro de la organización, con el fin de ganar eficiencia interna; la segunda se refiere a las relaciones con otras organizaciones; el tercero se ocupa de las autoridades en los que opera la multinacional, mientras que el último refiere a las cuestiones relacionadas con distintos actores del plano internacional. El área de la diplomacia internacional de negocios abarca las relaciones con los gobiernos locales, las ONG y las comunidades locales, mediante múltiples actores (Valderrey, 2016).

\section{La diplomacia internacional de negocios busca establecer y mantener relaciones positivas con los representantes de gobiernos extranjeros y las partes interesadas no gubernamentales}




\subsection{La diplomacia internacional de negocios como pieza clave en el proyecto de la nueva Ruta de la Seda}

La Nueva Ruta de la Seda abarca los tres ámbitos ya descritos de la diplomacia relacionada con aspectos económicos, pero requiere también de sus mecanismos tradicionales para solventar las situaciones más relevantes que se están produciendo. Empezaremos por discutir esta faceta, para a continuación referirnos al ámbito económico y de los negocios.

Desde el punto de vista de las relaciones internacionales, este gran proyecto puede también ser visto como parte de una triple diplomacia china -diplomacia de asociación, económica y financiera- que en conjunto persigue el rejuvenecimiento económico de China y el establecimiento de un nuevo orden financiero mundial Feng Zhang (2016). Otra visión, sin embargo, enfatiza que el objetivo de promover una mayor integración económica y el crecimiento regional mediante esta iniciativa, va más allá de cuestiones geoestratégicas, pues responde también a cuestiones de la política económica interna, lo que obliga al análisis de los vínculos entre el desarrollo interno de China y sus actividades externas (Gu, 2015).

Otro planteamiento enfatiza la combinación de nuevas ideas como el Sueño Chino, nuevas políticas de diplomacia y seguridad integral, nuevas instituciones, como por ejemplo el Banco de Infraestructura Asiática, y nuevos proyectos para construir lo que el presidente Xi denomina la "comunidad de destino compartido" (Callahan, 2016). En esta misma línea también se busca la manera en que China defina su identidad en relación a Estados Unidos y a otras potencias; por lo tanto, la estrategia detrás de la Nueva Ruta de la Seda es un asunto de la propia autodefinición de China (Larson, 2015).

Otro enfoque, otorga aún más valor al factor estadounidense y plantea que el origen de la renovada atención del gobierno chino hacia sus vecinos es resultado de una competencia por la hegemonía regional que ha tomado forma en las nuevas iniciativas de cooperación de seguridad regional y en las nuevas propuestas para la cooperación económica regional que se concretan en la Nueva Ruta de la Seda (Xinbo, 2016). Por último, se puede hacer incluir esta iniciativa dentro de la Gran Estrategia de China para fortalecer un orden multilateral más acorde a la promoción de sus propios intereses (Wang, 2015).

Además de los temas de alta diplomacia que se están suscitando a nivel comercial, existen en el trasfondo situaciones de balance de poder entre las diferentes potencias que tienen algún tipo de interés en la Nueva Ruta de la Seda. Como ya hemos señalado, Estados Unidos mantiene una postura antagónica al igual que países más cercanos a China, como es el caso de Japón y Corea del Sur. La India no puede ser tampoco indiferente y recientemente ha propiciado un acercamiento con el otro gigante asiático, aunque sin llegar a un involucramiento pleno en el proyecto. Rusia se muestra considerablemente más participativa, aunque no deja de tener recelos ante el avance hegemónico de su vecino en Asia central. Otros actores regionales se están alineando a uno y otro eje comercial, aunque en la mayoría de los casos sin cerrar las puertas a la relación comercial con ambas potencias.

En el trasfondo queda el potencial de conflicto en el mar de China Meridional, la protección de las rutas por las que se transporta una gran parte de los energéticos a nivel mundial y las tensiones ancestrales entre países del Sudeste Asiático y los de mayor proximidad con el Oriente Medio. En este sentido, la importancia de la Ruta Marítima del Siglo XXI de la Ruta de la Seda es claramente más importante, debido a la envergadura económica de las naciones que invo-
Se puede hacer incluir esta iniciativa dentro de la Gran Estrategia de China para fortalecer un orden multilateral más acorde a la promoción de sus propios intereses 
lucra, las alianzas presentes y la volatilidad de las políticas de relaciones exteriores, como es el reciente caso de Filipinas. Una demostración de la gran trascendencia del proyecto marítimo es que, cuando se proyecta en el mapa de Asia y África, este coincide en gran medida con lo que se ha dado en llamar el Collar de Perlas, o la cadena de bases chinas o de alianzas militares en el que ese país pudiera apoyarse ante posibles conflictos bélicos.

Mapa 2: Mapa del Collar de perlas y zonas de influencia naval de China

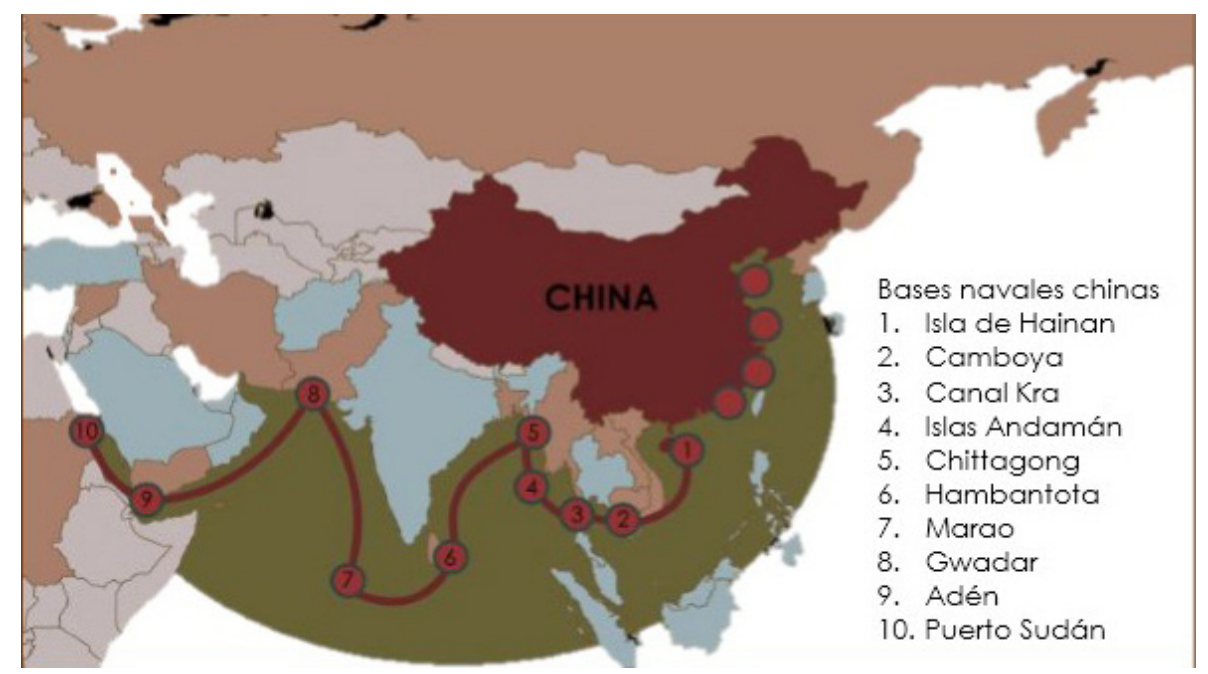

Fuente: http://il.wp.com/elordenmundial.com

Si bien las situaciones que han ido apareciendo hasta el momento han requerido de un enfoque tradicional de la diplomacia, esta es insuficiente ante los conflictos que comienzan a aparecer en la fase más operativa de los proyectos. En ese sentido, la diplomacia internacional de negocios es un complemento indispensable en la Nueva Ruta de la Seda, ya que permite un adecuado engranaje entre los objetivos estratégicos del gobierno chino y los operadores y beneficiarios directos de la implementación de la política económica internacional de ese país. En la medida en que las empresas involucradas en esta iniciativa sigan una adecuada diplomacia internacional de negocios podrán superar los abundantes obstáculos y desafíos que este proyecto representa.

Los obstáculos a los que se enfrentan tanto el gobierno como las empresas de China, son de diversa índole. Engloban los aspectos relacionados con las dificultades de realizar negocios en entornos diferentes, a los que hay que sumar los que se derivan de las enormes diferencias existentes en múltiples niveles entre los más de sesenta países participantes en la Nueva Ruta de la Seda. Algunos obstáculos son obvios, como la diferencia de regímenes políticos, los desequilibrios económicos entre las partes, la complejidad de sus relaciones históricas, o la diversidad étnica y cultural, pero otros son más sutiles. Entre estos últimos se incluyen las relaciones entre diferentes naciones y las consecuencias de que la nación se haya convertido en nueva potencia regional, lo que influye notablemente en algunos ámbitos que debieran pertenecer únicamente a los negocios. En efecto, a las tensas relaciones que China mantiene con algunos de sus vecinos, especialmente en el mar de China Meridional, hay que agregar las rivalidades históricas entre la India y Paquistán, o las dificultades de naciones dentro de naciones con predominio 
del Islam, las de países que fueron parte en su día de la extinta Unión Soviética, o simplemente de las derivadas de las dudas generadas por la dualidad entre los dos grandes ejes comerciales representados por el TPP y la Nueva Ruta de la Seda.

En la tabla 2, presentamos una clasificación de los principales obstáculos que van a surgir durante la fase operativa del proyecto, basada en la metodología desarrollada por The Economist Intelligence Unit para la evaluación de riesgos y desafíos que enfrentan las empresas participantes en este plan. La consultora emplea un exhaustivo análisis que mide el riesgo de operar en diferentes países utilizando los diez principales criterios que permiten cuantificar el impacto en la rentabilidad de negocio en cada uno de los países cubiertos otorgando una calificación general por país por medio de diferentes subindicadores por categoría.

\section{Tabla 2. Obstáculos para las empresas en la nueva Ruta de la Seda}

\begin{tabular}{|l|l|}
\hline OBSTÁCULOS & DESCRIPCIÓN \\
\hline Seguridad & Terrorismo, crimen organizado, extorsión, conflictos armados \\
\hline Estabilidad política & Tensiones internas, oposiciones, exceso de fuerza de autoridad \\
\hline Gobierno & Burocracia, corrupción, derechos humanos \\
\hline Legal y regulatorio & Procesos judiciales, control de precios, propiedad intelectual, prácticas desleales \\
\hline Macroeconómico & Recesiones, tasas de cambio, riesgo de recesión, inestabilidad económica \\
\hline Comercio internacional & Crisis financiera, tarifas arancelarias, embargos \\
\hline Financiero & Devaluación, deudas, falta de liquidez en mercado accionario \\
\hline Política fiscal & Impuestos discriminatorios, retorno de impuestos, tasa impositiva corporativa \\
\hline Mercado laboral & Huelgas, sindicatos, poca remuneración \\
\hline Infraestructura & Redes de comunicación y transporte terrestre, tecnología, infraestructura portuaria \\
\hline
\end{tabular}

Fuente: Prospects and challenges on China's 'one belt, one road': a risk assessment report, Economist Intelligence Unit (2015)

En el caso de la Nueva Ruta de la Seda, todos los factores de riesgos señalados están presentes, en mayor o menor medida, y en diferentes países. La magnitud del proyecto y el número de países, agravan el riesgo en su agregado, especialmente cuando se considera que las naciones participantes no tienen vínculos ideológicos o de algún otro tipo, que les permita actuar al unísono, al sentirse parte de un destino común. Los nexos entre estos países son prevalentemente de conveniencia, especialmente derivada del oportunismo de ser partícipes de la ayuda económica china, por el momento sumamente generosa. En ocasiones se ha equiparado a este proyecto con el Plan Marshall, pero las diferencias son notorias y difícilmente se sostiene dicha comparación (Esteban y Otero-Iglesias, 2016).

\subsection{Dimensión económica y sectores beneficiados}

Las oportunidades ya presentes en la Nueva Ruta de la Seda, pueden verse impulsadas en el marco de una coyuntura en la que el gobierno chino se está viendo forzado a adoptar medidas drásticas, con las que pueda paliar las consecuencias de la desaceleración de su economía. Conviene recordar que, pese a los éxitos económicos que en tan solo 30 años transformaron a un país 
agrícola con todo tipo de rezagos en potencia económica global (Cheung y Lee, 2016), la realidad actual está cambiando velozmente. El modelo de inversión y producción al interior de China y su vocación exportadora hacia los mercados desarrollados le permitieron convertirse en la segunda mayor economía del mundo, no tan distante de los Estados Unidos. Sin embargo, la necesidad de mantener sus indicadores y los objetivos de sus metas previamente marcadas, puede empujar a la élite burocrática a la búsqueda de nuevas alternativas para sostener el crecimiento económico.

Las grandes inversiones requieren ser publicitadas por el Partido Comunista Chino, en el intento de ensalzar los nuevos logros del país y ciertamente de sus dirigentes. Sin embargo, a las tradicionales dificultades para obtener cifras y estadísticas confiables en ese país, debe agregarse la multiplicidad de proyectos asociados a la Nueva Ruta de la Seda, con las consiguientes ambigüedades en cuanto a su impacto específico en esta iniciativa. Las cantidades totales estimadas varían considerablemente conforme a diferentes fuentes, aunque en todos los casos, las cifras monetarias son de enorme magnitud. Un reporte de la firma de consultoría PwC estima que hasta comienzos del 2016 ya se habían invertido 250 billones de dólares ${ }^{2}$ en diferentes proyectos directamente relacionados con esta iniciativa (Van der Leer y Yau, 2016). Los autores del reporte propugnan que la Nueva Ruta de la Seda es en realidad la suma de diferentes proyectos entrelazados entre sí, por lo que despierta dudas sobre si pertenecen a una única visión, a una sola estrategia o son tan solo parte de un eslogan político.

Los montos que se alcanzarán en este proyecto a corto y medio plazo se estiman en trillones de dólares, mientras que las posibles inversiones que se generen a largo plazo son demasiado difíciles de cuantificar en este momento. Las cifras más fiables son las que anuncian los organismos oficiales directamente involucrados en los proyectos; así, por ejemplo, el China Development Bank se declara preparado para intervenir con cerca de 900 billones de dólares, mientras que el Asia Infrastructure Investment Bank tiene preparada una importante cantidad para respaldar el proyecto. Otras entidades financieras tienen también considerado el apoyo para satisfacer los enormes requerimientos financieros y el propio gobierno dotó al proyecto con un fondo de aproximadamente 40 billones de dólares, mediante el New Silk Road Fund.

Independientemente de cuáles sean las cantidades finales de inversión, es evidente la derrama económica que se está generando para las empresas involucradas; en un inicio se pensó que las más beneficiadas serían las que proporcionan servicios relacionados con la construcción y el desarrollo de infraestructuras, pero la bonanza ya está extendiéndose a múltiples sectores, como presentamos en la tabla 3.

Tabla 3. Principales sectores beneficiados por la nueva Ruta de la Seda

\begin{tabular}{|l|l|l|l|}
\hline Sector & Características & Ejemplos de proyectos & $\begin{array}{l}\text { Principales } \\
\text { inversionistas }\end{array}$ \\
\hline Infraestructura & $\begin{array}{l}\text { Construcción y desarrollo en } \\
\text { puertos de Asia central y del este }\end{array}$ & $\begin{array}{l}\text { Construcción de mega embarcaderos en } \\
\text { el Puerto de Singapur }\end{array}$ & $\begin{array}{l}\text { China Harbour } \\
\text { Engineering Company } \\
\text { (CHEC) }\end{array}$ \\
\hline Transporte & $\begin{array}{l}\text { Inversión en trenes de alta } \\
\text { velocidad para el Sureste Asiático }\end{array}$ & $\begin{array}{l}\text { Tren de alta velocidad Sino-Thai } \\
\text { Railway para conectar Tailandia, China } \\
\text { y Laos }\end{array}$ & $\begin{array}{l}\text { China Railway } \\
\text { Corporation }\end{array}$ \\
\hline Energía & $\begin{array}{l}\text { Construcción de nuevas plantas de } \\
\text { generación de energías sostenibles }\end{array}$ & $\begin{array}{l}\text { Planta de energía solar más grande del } \\
\text { mundo en Bahawalpur, Paquistán }\end{array}$ & $\begin{array}{l}\text { Powerchina Resources } \\
\text { Limited }\end{array}$ \\
\hline
\end{tabular}

2 Las cifras reportadas en dólares en este documento se refieren a dólares americanos. Los billones se representan como miles de millones de esa moneda, mientras que cada trillón de dólares corresponde a mil billones. 


\begin{tabular}{|l|l|l|l|}
\hline Turismo & $\begin{array}{l}\text { Inversión en promoción turística } \\
\text { para sitios patrimonio de la } \\
\text { Humanidad }\end{array}$ & $\begin{array}{l}\text { Desarrollos en China y Sureste Asiático } \\
\text { con UNESCO }\end{array}$ & $\begin{array}{l}\text { China Road and Bridge } \\
\text { Corporation }\end{array}$ \\
\hline Manufactura & $\begin{array}{l}\text { Experiencia china en manufactura, } \\
\text { trenes de alta velocidad, energía } \\
\text { nuclear }\end{array}$ & $\begin{array}{l}\text { Creación de Corredor Económico China- } \\
\text { Paquistán }\end{array}$ & $\begin{array}{l}\text { China State Construction } \\
\text { Engineering Corporation }\end{array}$ \\
\hline Finanzas & $\begin{array}{l}\text { Demanda de bonos, seguros, } \\
\text { administración de cartera de } \\
\text { activos }\end{array}$ & $\begin{array}{l}\text { Creación de fondos de inversión para } \\
\text { financiar proyectos }\end{array}$ & $\begin{array}{l}\text { Infrastructure Financing } \\
\text { Facilitating Office con: } \\
\text { HSBC, GE, KPMG, etc. }\end{array}$ \\
\hline
\end{tabular}

Fuente: elaboración propia, adaptado de Ernst \& Young, "Navigating the Belt and Road", (2015).

\subsection{Las empresas en la nueva Ruta de la Seda}

En la tabla 3 señalamos los sectores más beneficiados en este plan, lo que sin embargo no significa que las oportunidades que se presentan puedan traducirse fácilmente en prósperos negocios. Puesto que las primeras empresas que han intervenido han sido, por lo general, paraestatales del gobierno chino, los criterios de negocio han quedado comúnmente subordinados a motivaciones geoestratégicas. La información de los resultados de tales operaciones no está disponible, como no lo está tampoco una lista que incluya a las empresas involucradas en esta iniciativa. Se sabe que hay un gran número de ellas, pero también hay un número creciente de ellas que parecen ser parte de la Nueva Ruta de la Seda, cuando en realidad tan solo intentan beneficiarse ganando notoriedad o posiblemente apoyos oficiales (Fok, 2016).

Al estudiar el perfil de las empresas chinas involucradas en esta iniciativa, es necesario entender que la composición de la inversión de China en el extranjero está evolucionando rápidamente, con nuevos actores apareciendo en escena, que reemplazan paulatinamente a las empresas estatales o con fuerte dependencia del Gobierno y subordinadas a intereses geoestratégicos (Valderrey y Montoya, 2016). Nuevas empresas están iniciando su proceso de internacionalización, alcanzando un porcentaje que se estima cercano al $25 \%$ de la inversión total no financiera, ofreciendo diversidad en su oferta y aportando nuevos desarrollos tecnológicos (Niu, 2015, p. 43). Los entes considerados demasiado próximos al Gobierno o las instituciones para la promoción de las exportaciones, como el China Development Bank o el Export and Import Bank of China, están cobijando a nuevas empresas, que claramente gozan de apoyo financiero en condiciones mejores a las que ofrecería el mercado. Además de las organizaciones citadas, merece especial consideración el caso del China Council for the Promotion of International Trade, puesto que interviene como agente intermediario entre el gobierno y las empresas a las que se apoya (Dussel y Armony, 2015).

En fechas recientes, las oportunidades de negocio se están abriendo paulatinamente a empresas de otros países, incluyendo de naciones que no están directamente vinculadas a esta iniciativa. Entre estas destacan las empresas europeas que ya han participado en proyectos de inversión dentro de la misma China, como en el caso de la construcción de la presa de las Tres Gargantas, las que aportan soluciones de ingeniería o tecnologías no disponibles hasta el momento en ese país, o aquellas que mantienen inversiones directas en China. Un ejemplo es el de la empresa francesa Alstom, que está reconvirtiendo algunas de las turbinas gigantes construidas para dicho proyecto, pero que ahora serán revendidas en terceros países, mediante proyectos de coinversión con empresas chinas (Hornby, 2016). El gobierno chino en su momento impulsó con gran ímpetu la internacionalización de sus empresas, bajo la política conocida como Going Abroad (salir al exterior), pero últimamente a tales esfuerzos se están aunando 
las solicitudes más claras y directas para atraer las aportaciones de capital por parte de otros países o de empresas.

Las medidas de inclusión de la iniciativa privada pueden también interpretarse como una estrategia para compartir el control del proyecto, bien sea debido a limitaciones en la disponibilidad de recursos o para hacer frente al recelo que despierta el excesivo poder y protagonismo de China. Al efecto, el gobierno continúa emitiendo reglas a las que deben someterse las empresas que inviertan en la Nueva Ruta de la Seda, intentando limitar su propia intervención o limitarla a establecer y operar procedimientos de control. Ejemplo de ello es el énfasis en el cumplimiento de principios que regulan el impacto ambiental y la responsabilidad social (Minghui, 2016).

En la medida que la dirección de los proyectos recaiga en menor medida en el gobierno, se aumentará el número de actores involucrados, lo que entendemos requerirá en mayor medida el uso de la diplomacia internacional de negocios por parte de las empresas participantes. Hasta ahora, el uso de las herramientas que brinda este tipo de diplomacia ha sido más limitado, generalmente con acciones demasiado locales y no en aquellas en las que las empresas chinas puedan resolver problemas de gran envergadura sin necesidad de recurrir a los mecanismos tradicionales que brinda la diplomacia tradicional.

\section{Conclusiones}

En este artículo hemos revisado los supuestos teóricos de la interdependencia compleja de Keohane y Nye (1973, 1998, 2000, 2001), que afirman que la visión estatocéntrica del escenario internacional es insuficiente para comprender la actual dinámica de interacciones entre actores estatales y no estatales. Esta dinámica es la base desde la que se diseña y conduce la agenda internacional, mientras que los intereses nacionales son el resultado de un proceso interno de negociación entre diversos actores que inciden en la política exterior de los Estados. Por lo tanto, en la manera que se concibe y aplica la política internacional, esta es el resultado de una interdependencia compleja.

Entendiendo que la Nueva Ruta de la Seda representa un ejemplo paradigmático de los postulados de dicha teoría, hemos descrito la necesidad de incluir en el análisis de esta iniciativa, la multiplicidad de actores y el uso de nuevas formas de diplomacia. Como hemos mencionado previamente, mediante este proyecto, el Gobierno chino persigue diferentes propósitos, entre los que se encuentra garantizar el crecimiento económico para la siguiente década. Este objetivo es prioritario para el Gobierno, pero también beneficia y genera rentabilidad a las empresas participantes. Sin embargo, estos proyectos se originan en un contexto específico en la que las empresas internacionales negocian, promueven e interactúan con gobiernos extranjeros y con diferentes públicos locales.

Creemos que este espacio es el escenario propicio para la diplomacia internacional de negocios, puesto que aporta mecanismos de resolución de conflictos más idóneos ante la complejidad de intereses interdependientes. Defendemos, por lo tanto, la validez de la diplomacia internacional de negocios como elemento complementario que puede coadyuvar al éxito de este proyecto y de aquellos que presenten un alto grado de complejidad, especialmente en la complementariedad de Estado-nación con empresa privada, cuando esta opera más allá de su mercado local.

\section{La visión} estatocéntrica del escenario internacional es insuficiente para comprender la actual dinámica de interacciones entre actores estatales y no estatales 


\section{Referencias}

Abbas, A. (2009). Managers and diplomacy. International Journal of Commerce and Management, 19(4), 256-258.

Beeson, M., \& Li, F. (2016). China’s Place in Regional and Global Governance: A New World Comes into View. Global Policy. doi:10.1111/1758-5899.12348

Berridge, G. R. (2002). Diplomacy: Theory and Practice. Basingstoke, Hampshire: Palgrave.

Bhoothalingam, R. (2016). The Silk Road as a Global Brand. China Report, 52(1), 45-52. doi: $10.1177 / 0009445515613869$

Callahan, W. A. (2016). China's "Asia Dream": The Belt Road Initiative and the new regional order. Asian Journal of Comparative Politics. doi: 10.1177/2057891116647806

Cheung, F., \& Lee, A. (2016). A brilliant plan: One Belt, One Road. Hong Kong: CLSA. Recuperado de: https:/www.clsa.com/special/onebeltoneroad/

Der Derian, J. (2001). Diplomacy. En The Oxford Companion to Politics of the World. Oxford: Oxford University Press.

Dussel, E., \& Armony, A. (2015). Beyond Raw Materials Who are the Actors in the Latin America and Caribbean-China Relationship? Buenos Aires: Red Académica de América Latina y el Caribe sobre China y Universidad de Pittsburgh.

Economist Intelligence Unit. (2015). Prospects and challenges on China's 'one belt, one road': a risk assessment report. Recuperado de: https://staticl.squarespace.com/ static/529fcf02e4b0aa09f5b7ff67/t/554c49cee4b06fc215162cb4/1431062990726/ One+Belt,+One+Road.pdf

Esteban, M., \& Otero-Iglesias, M. (2015). ¿Qué podemos esperar de la nueva Ruta de la Seda y del Banco Asiático de Inversión en Infraestructuras liderados por China? Real Instituto Elcano. Recuperado de: http://www.realinstitutoelcano.org/wps/portal/web/rielcano_es/ contenido?WCM_GLOBAL_CONTEXT=/elcano/elcano_es/zonas_es/asia-pacifico/ ari18-2015-esteban-otero-que-podemos-esperar-nueva-ruta-de-la-seda-y-banco-asiatico-de-inversion-en-infraestructuras-liderados-por-china

Ferdinand, P. (2016). Westward ho-the China dream and 'one belt, one road': Chinese foreign policy under Xi Jinping. International Affairs, 92(1), 941-957. doi: 10.1111/14682346.12660

Fok, A. (2016, Junio 15). One Belt, Many Companies: The great bandwagoning. Harbor Times. Recuperado de: http://harbourtimes.com/2016/06/15/one-belt-many-companies-the-great-bandwagoning/

Gu, J. (2015). China's New Silk Road to Development Cooperation: Opportunities and Challenges. Nueva York: United Nations University Centre for Policy Research. Recuperado de: http://i.unu.edu/media/cpr.unu.edu/attachment/1803/UNUCPR_ChinasNewSilkRoad_Gu_.pdf

Hansen, V. (2015). The Silk Road: A New History. Oxford: Oxford University Press. 
Hornby, L. (2016, Mayo 25). China seeks foreign investors for One Belt, One Road push. Financial Times. Obtenido de: https://www.ft.com/content/b04c0800-2246-11e6-aa98dble01fabc0c

Keohane, R. (1984). After Hegemony: Cooperation and Discord in the World Political Economy. Princeton, NJ: Princeton University Press.

Keohane, R., \& Nye, J. (1973). Transnational Relations and World Politics: A Conclusion. En R. Keohane y J. Nye (Eds.), Transnational Relations and World Politics (pp. 371-398). Cambridge, Massachusetts: Cambridge University Press.

Keohane, R., \& Nye, J. (1998). Power and interdependence in the information age. Foreign Affairs, 77(5), 81-94.

Keohane, R., \& Nye, J. (2000). Globalization: What's New? What's Not? (And So What?). Foreign Policy, 118, 104-1 19. doi: 10.2307/1149673

Keohane, R., \& Nye, J. (2001). Power and Interdependence. Nueva York: Longman.

La Porte, T. (2016). Influencia de los actores internacionales no-estatales en las estrategias diplomáticas: consideraciones desde la comunicación pública. Comillas Journal of International Relations, 6, 28-39.

Larson, D. W. (2015). Will China be a New Type of Great Power? Chinese Journal of International Politics, 8(4), 323-348. doi: 10.1093/cjip/pov010

Lee, D., \& Ruël, H. (2012). Introduction: Commercial Diplomacy and International Business: Merging International Business and International Relations. En D. Lee \& H. Ruë (Eds.), Commercial Diplomacy and International Business: A Conceptual and Empirical Exploration (pp. 13-19). Bingley, R.U.: Emerald Group Publishing Limited.

Lee, D. (2016, agosto 18). One belt, one road not 'by china, for china'. The Business Times. Recuperado de: http://0-search.proquest.com.millenium.itesm.mx/docview/181228078 8 ?accountid $=41938$

Lo, C. (2015). China's Silk Road strategy: the opportunities and pitfalls of 'killing three birds with one stone'. The International Economy, 29(4), 54-71.

Menzies, G. (2008). 1421: The Year China Discovered America. New York: Harper Collins Publisher.

Minghui, H. (2016, Julio 11). China's First Chinese-Invested Company Social Responsibility Roadmap for the One Belt, One Road Initiative Launched. China Federation of Industrial Economics. Disponible en: http://www.prnewswire.com/news-releases/chinas-firstchinese-invested-company-social-responsibility-roadmap-for-the-one-belt-one-road-initiative-launched-300297009.html

Moons, S., \& van Bergeijk, P. (2016). Does Economic Diplomacy Work? A Meta-analysis of Its Impact on Trade and Investment. The World Economy. doi: 10.1111/twec.12392

Naqvi, M. (2015, Nov 26). What exactly is 'one belt, one road'? The Financial Daily. Recuperado de: http://0-search.proquest.com.millenium.itesm.mx/docview/1736567642?accou ntid=41938 
National Development and Reform Commission. (2015). Action plan on the China-proposed Belt and Road Initiative. Recuperado de: http:/www.chinagoabroad.com/zh/article/action-plan-on-the-china-proposed-belt-and-road-initiative

Niu, H. (2015). A New Era of China-Latin America Relations. Inter-American Development Bank. Recuperado de: http://www 19.iadb.org/intal/intalcdi/PE/2016/16033.pdf

Olier, E. (2016). Los Ejes del Poder. Madrid: Pearson.

Rasmussen, S. B. (2016). La diplomacia de la UE como una superposición cuántica: ¿̇hacia un marco conceptual para investigaciones multidisciplinares de un fenómeno de pluralización? Comillas Journal of International Relations, 6(1), 13-27.

Ruël, H. (2013). Diplomacy means business. Windesheimreeks kennis en onderzoek, 46. Disponible en: http://www.windesheim.nl

Saner, R., Yiu, L., \& Sondergaard, M. (2000). Business diplomacy management: a core competency for global companies. Academy of Management Perspectives, 14(1), 80-92. doi: 10.5465/AME.2000.2909841

Van der Leer, Y., \& Yau, J. (2016). China's new silk route. The long and winding road. PwC. Disponible en: http://www.pwc.com/gx/en/issues/high-growth-markets/publications/ china-new-silk-route.html

Valderrey, F. J. (2016). International Business Diplomacy: Lessons from Latin America. En M. A. Khan (Ed.), Multinational Enterprise Management Strategies in Developing Countries (pp. 295-315). Hershey, PA: IGI Global.

Valderrey, F., \& Montoya, M. A. (2016). China to Finance Infrastructure in Latin America. Network Industries Quarterly, 18(2).

Wang, Z. (2015). Europe in China's Incremental Intercontinentalism. Asian Politics E Policy, 7(1), 131-144. doi:10.1111/aspp. 12165

Wolters, T. (2012). Business diplomacy in multinational corporations: An exploratory qualitative study. (Master's thesis). University of Twente, Enschede, Netherlands. Recuperado de: http://purl.utwente.nl/essays/62485

Xinbo, W. (2016). Cooperation, competition and shaping the outlook: The United States and China's neighborhood diplomacy. International Affairs, 92(4), 849-867. doi: $10.1111 / 1468$

Zhang, F. (2016). China as a Global Force. Asia and the Pacific Policy Studies, 3(1), 120-128. doi: 10.1002/app5.115

Zhang, Y. (2016), Introduction: Dynamism and contention: understanding Chinese foreign policy under Xi Jinping. International Affairs, 92(4), 769-772. doi: 10.1111/14682346.12661

Zhao, K. (2015). The Motivation behind China's Public Diplomacy. Chinese Journal of International Politics, 8(2), 167-196. doi: 10.1093/cjip/pov005 